\title{
GW150914: The Advanced LIGO Detectors in the Era of First Discoveries
}

\author{
B. P. Abbott et al. \\ (LIGO Scientific Collaboration and Virgo Collaboration)
}

(Received 15 February 2016; published 31 March 2016)

\begin{abstract}
Following a major upgrade, the two advanced detectors of the Laser Interferometer Gravitational-wave Observatory (LIGO) held their first observation run between September 2015 and January 2016. With a strain sensitivity of $10^{-23} / \sqrt{\mathrm{Hz}}$ at $100 \mathrm{~Hz}$, the product of observable volume and measurement time exceeded that of all previous runs within the first 16 days of coincident observation. On September 14, 2015, the Advanced LIGO detectors observed a transient gravitational-wave signal determined to be the coalescence of two black holes [B. P. Abbott et al., Phys. Rev. Lett. 116, 061102 (2016)], launching the era of gravitational-wave astronomy. The event, GW150914, was observed with a combined signal-to-noise ratio of 24 in coincidence by the two detectors. Here, we present the main features of the detectors that enabled this observation. At full sensitivity, the Advanced LIGO detectors are designed to deliver another factor of 3 improvement in the signal-to-noise ratio for binary black hole systems similar in mass to GW150914.
\end{abstract}

DOI: 10.1103/PhysRevLett.116.131103

Introduction.-On September 14, 2015, both Advanced LIGO detectors in the USA, H1 in Hanford, Washington and L1 in Livingston, Lousiana, made the first direct measurement of gravitational waves [1]. The event, GW150914, was determined to be the merger of two black holes, with masses of 36 and $29 M_{\odot}$, into a black hole of approximately $62 M_{\odot}$ [2]. 3.0 solar masses of energy $\left(\simeq 5.4 \times 10^{47} \mathrm{~J}\right)$ were radiated in gravitational waves. The gravitational waves from this event, which occurred at a distance of $\simeq 410 \mathrm{Mpc} \simeq 1.3 \times 10^{9}$ light years, changed the separation between the test masses by $\simeq 4 \times 10^{-18} \mathrm{~m}$, about one 200th of a proton radius.

The Advanced LIGO detectors, multikilometer Michelson-based interferometers [3], came online in September 2015, after a major upgrade targeting a factor of 10 sensitivity improvement over initial detectors [4,5]. While not yet at design sensitivity during their first observation run, they have already exceeded the strain sensitivity of the initial detectors across the entire frequency band, significantly surpassing the past discovery potential. This Letter describes the Advanced LIGO detectors, as well as their current and final design sensitivity, at the inception of gravitational-wave astronomy [6-10].

Astrophysical reach.-In general relativity, a gravitational wave far from the source can be approximated as a time-dependent perturbation of the space-time metric, expressed as a pair of dimensionless strain polarizations, $h_{+}$and $h_{\times}$[11]. An interferometric gravitational-wave detector acts as a transducer to convert these space-time perturbations into a measurable signal [12]. The interferometer mirrors act as "freely falling" test masses. Advanced LIGO measures linear differential displacement along the arms which is proportional to the gravitational-wave

*Full author list given at the end of the article. strain amplitude. We define the differential displacement as $\Delta L=\delta L_{x}-\delta L_{y}$, where $L_{x}=L_{y}=L$ are the lengths of two orthogonal interferometer arms. The gravitationalwave strain and the interferometer displacement are related through the simple equation $\Delta L=h L$, where $h$ is a linear combination of $h_{+}$and $h_{\times}$.

The tiny displacements induced by astrophysical events demand that the interferometer mirrors be free from environmental disturbances and require a highly sensitive interferometric transducer-designed to be limited only by disturbances arising from fundamental physics considerations. Since the interferometer response to displacement, or equivalently gravitational-wave strain, is frequency dependent, it is necessary to represent the limiting detector noises as functions of frequency normalized by the interferometer response.

The left panel of Fig. 1 shows the amplitude spectral density of the total strain noise in units of strain per $\sqrt{\mathrm{Hz}}$ during the first observation run (O1 run) and, for comparison, during the final science run of the initial LIGO detectors (S6 run). In the detectors' most sensitive frequency band between 100 and $300 \mathrm{~Hz}$, the $\mathrm{O} 1$ strain noise is 3 to 4 times lower than that achieved in the S6 run. At $50 \mathrm{~Hz}$, the improvement is about a factor of 30 .

The right panel of Fig. 1 shows the single detector signalto-noise ratio (SNR) for an optimally oriented compact binary system consisting of two $30 M_{\odot}$ black holes as a function of redshift $z$ and for different interferometer configurations. The observed strain amplitude is largest for a source whose orbital plane is parallel to the plane of the detector and is located straight above or below; we refer to such a source as optimally oriented. The SNR is computed in the frequency domain [14] using standard cosmology [15] and phenomenological waveforms which account for inspiral, merger, and ringdown, but not spins [16]. 

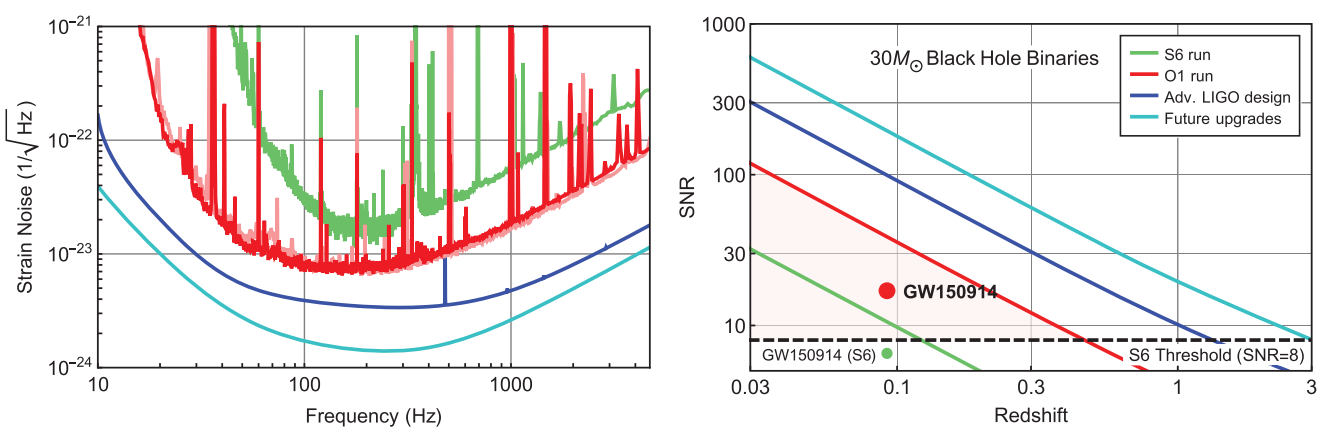

FIG. 1. The left plot shows the strain sensitivity during the first observation run (O1) of the Advanced LIGO detectors and during the last science run (S6) of the initial LIGO detectors. The O1 strain noise curve is shown for H1 (dark red) and L1 (light red); the two detectors have similar performance. The Advanced LIGO design sensitivity, as well as a possible future upgrade [13], are shown to highlight the discovery potential in the coming years. The right plot shows the single detector signal-to-noise ratio (SNR) under optimal orientation as a function of redshift $z$ - for two merging black holes with mass $30 M_{\odot}$ each. GW150914 was not optimally orientated and was detected with a single detector SNR of 13 to 20 at $z=0.09$; this event would not have been seen in S6.

A Michelson interferometer lacks good directional sensitivity to gravitational waves. The antenna pattern covers approximately half the sky, both above and beneath the plane of the detector. Moreover, the antenna patterns of the two LIGO detectors are aligned to maximize the coincident detection of gravitational-wave signals, constrained to the $10 \mathrm{~ms}$ intersite propagation time. The coincidence constraint substantially rejects non-Gaussian noise and vetoes local transients.

The observed strain amplitude is inversely proportional to the luminosity distance. For small redshifts, $z<1$, the observable volume, and, thus, the detection rate, grows as the cube of the detector sensitivity. The number of detected events is expected to scale with the product of observing volume and observing time. Between September 12, 2015 and October 20, 2015, the H1 and L1 detectors had a duty cycle of $70 \%$ and $55 \%$, respectively, while the observing time in coincidence was $48 \%$. After data quality processing [17], 16 days of data were analyzed around GW150914, resulting in a time-volume product of $0.1 \mathrm{Gpc}^{3} \mathrm{yr}$ for binary black hole systems with masses similar to GW150914 [18].

The displacement measurement.-The current generation of advanced detectors uses two pairs of test masses as coordinate reference points to precisely measure the distortion of the space-time between them. A pair of input and end test masses is located in each of the two arms of a Michelson laser interferometer, as shown in Fig. 2. The Advanced LIGO test masses are very pure and homogeneous fused silica mirrors of $34 \mathrm{~cm}$ diameter, $20 \mathrm{~cm}$ thickness, and $40 \mathrm{~kg}$ mass.

It is critical that the test masses be free from sources of displacement noise, such as environmental disturbances from seismic noise, or thermally driven motion. These noise sources are most relevant at frequencies below $100 \mathrm{~Hz}$, while shot noise of the optical readout is dominant at high frequencies. Figure 3 shows the measured displacement noise of Advanced LIGO during the first observing run, together with the major individual contributions, as discussed below.

To reduce the effects of ground vibrations, the test masses are suspended by multistage pendulums [20], thus,
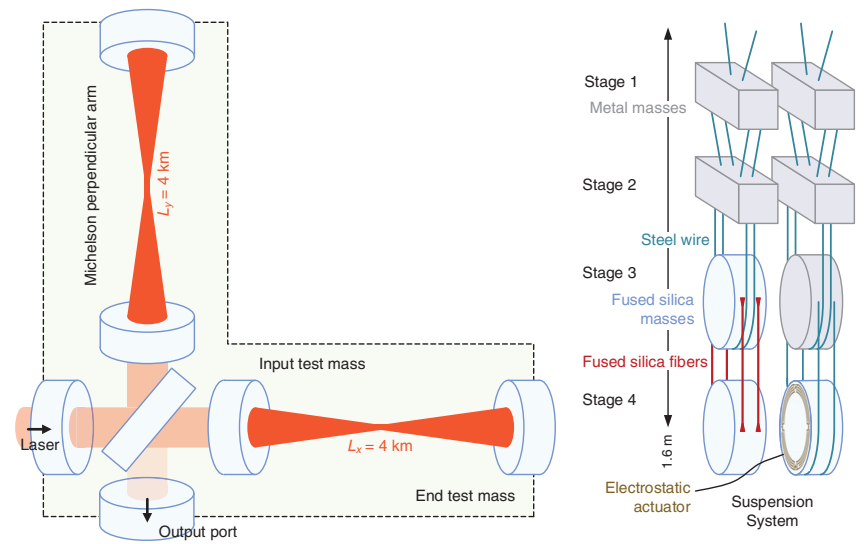

FIG. 2. Interferometer configuration and test mass setup. Each arm of the Michelson interferometer includes two suspended test masses. The two test masses are placed $4 \mathrm{~km}$ apart and form an optical resonator with a gain of 300. The suspension system is shown on the right, each test mass is at the bottom of a quadruple pendulum. It provides high isolation above the resonance frequencies which range from 0.4 to $13 \mathrm{~Hz}$. The test mass is attached to the penultimate mass through fused silica fibers providing a high mechanical quality factor which lowers the thermal noise. The other stages use steel wire. The attachment point to the seismic isolation system as well as stages 1 and 2 implement cantilever springs for vertical isolation. Each test mass is accompanied by its own reaction chain to minimize actuation noise. Coil actuators are mounted to the upper stages of the reaction chain, and an electrostatic actuator is implemented at the bottom stage. Shown on the left are the other optics of the Michelson interferometer with the beam splitter and the perpendicular arm. The two optics at the interferometer input and output port comprise the coupled resonator system which amplifies the response of the optical transducer. 


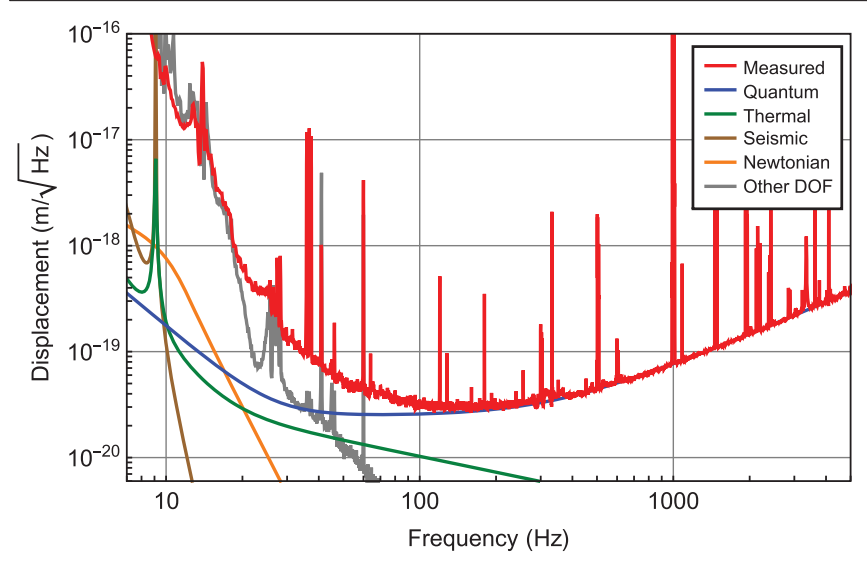

FIG. 3. The displacement sensitivity of the Advanced LIGO detector in Hanford during the first observation run $\mathrm{O} 1$; the Livingston detector has a similar sensitivity, as shown in Fig. 1. The sum of all known noise sources accounts for most of the observed noise with the exception of the frequency band between 20 and $100 \mathrm{~Hz}$. This will be the focus of future commissioning to full sensitivity. The quantum noise includes both shot noise and radiation pressure noise. Thermal noise includes terms due to the suspensions, the test masses, and the coatings. Seismic noise is the ground displacement attenuated through the seismic isolation system and the suspensions. Cross couplings from the autoalignment system and from the auxiliary lengths are combined into the trace labeled "other DOF" (degrees of freedom). Newtonian gravitational noise is estimated from density perturbations due to surface ground motion. The strong line features are due to the violin modes of the suspension wires, the roll and bounce modes of the suspensions, the AC power line and its harmonics, and the calibration lines. Not shown are numerous noise sources that do not contribute significantly-such as laser frequency, intensity and beam jitter noise, sensor and actuation noise, and Rayleigh scattering by the residual gas [19].

acting as free masses well above the lowest pendulum resonance frequency of $0.4 \mathrm{~Hz}$. Monolithic fused silica fibers [21] are incorporated at the bottom stage to decrease suspension thermal noise [22], which limits the useful frequencies to $10 \mathrm{~Hz}$ and above. The Advanced LIGO test masses require about 10 orders of magnitude suppression of ground motion above $10 \mathrm{~Hz}$. The multistage pendulum system attenuates the ground motion by 7 orders of magnitude. It is mounted on an actively controlled seismic isolation platform which provides 3 orders of magnitude of isolation of its own [23,24]. Moreover, these platforms are used to reduce the very large displacements produced by tidal motion and microseismic activity. Tidal forces can produce displacements up to several hundred $\mu \mathrm{m}$ over a multikilometer baseline on time scales of hours. The dominant microseismic activity is driven by ocean waves. The resulting ground motion can be as large as several $\mu \mathrm{m}$ at frequencies around $0.15 \mathrm{~Hz}$ - even far inland.

The entire test mass assembly including the suspension system and part of the seismic isolation system resides inside an ultrahigh vacuum system, with pressures typically below $1 \mu \mathrm{Pa}$ over the $10000 \mathrm{~m}^{3}$ volume, to prevent acoustic shorting of the seismic isolation systems and to reduce Rayleigh scattering in the optical readout.

The test masses are also susceptible to changes in the local gravitational field caused by changing mass distributions in their vicinity. While not limiting presently, at design sensitivity, this time-dependent Newtonian noise source possibly becomes relevant below $20 \mathrm{~Hz}$, and might require active cancellation $[25,26]$.

Thermally driven motion is another important source of displacement noise. It includes the Brownian motion of the suspension system [27] as well as the test masses [28] and mechanical loss in the mirror optical coatings [29]. The mirror coatings, a dielectric multilayer of silica and titania-doped tantala [30,31], were developed to provide the required high reflectivity while minimizing coating thermal noise [32-34]; at design, it will eventually limit the sensitivity around $60 \mathrm{~Hz}$ [3].

The predicted levels for seismic, thermal, and Newtonian noise sources are summarized in Fig. 3 and compared to the total measured displacement noise. They are currently not limiting the sensitivity due to the presence of other technical noise sources, summarized in the next section and detailed in Ref. [19].

Quantum noise in the interferometer arises from the discrete nature of photons and their Poisson-distributed arrival rate [35-37]. The momentum transfer of individual photons hitting a test mass gives rise to radiation pressure noise. Quantum radiation pressure noise scales as $1 / m f^{2}$, where $m$ is the mass of the mirror and $f$ the frequency, and therefore, it is most significant at lower frequencies.

Photon shot noise arises from statistical fluctuations in the photon arrival time at the interferometer output, and it is a fundamental limit of the transducer in sensing changes of the differential arm length. The importance of shot noise decreases as the inverse square root of the laser power circulating in the interferometer arms. During the first observing run, Advanced LIGO was operating with $100 \mathrm{~kW}$ of circulating laser power. The corresponding quantum noise curve, comprising both low frequency radiation pressure noise and high frequency shot noise, is shown in Fig. 3; it is limiting at frequencies above $70 \mathrm{~Hz}$. In the upcoming years, we plan to increase the circulating laser power up to $750 \mathrm{~kW}$ and, thus, reduce the shot noise contribution.

Coincident detection between the two LIGO observatories is used to reject transient environmental disturbances. Both observatory sites deploy seismometers, accelerometers, microphones, magnetometers, radio receivers, weather sensors, ac-power line monitors, and a cosmic ray detector for vetoes and characterization of couplings [38].

Interferometric transducer.-The Advanced LIGO detector uses a modified Michelson laser interferometer to translate strain into an optical phase shift [3]. Similar to an electromagnetic receiver, the optimal antenna length for 
a gravitational-wave detector is a quarter wavelength. For a gravitational wave at $100 \mathrm{~Hz}$, this is $750 \mathrm{~km}$. The Advanced LIGO interferometer arms are $4 \mathrm{~km}$ long and employ an optical resonator between the input and end test masses that multiplies the physical length by the effective number of round-trips of the light. However, the physical length cannot be arbitrarily short because test mass displacement noises are multiplied by the same factor.

The output port of the Michelson interferometer is held at an offset from a dark fringe, resulting in a small amount of light leaving the output port [39]. A differential optical phase shift will then decrease or increase the amount of light, depending on which interferometer arm is momentarily stretched or squeezed by a passing gravitational wave. This light signal is measured by a photodetector, digitized, and calibrated [40] before being sent to the analysis pipelines [41,42].

The calibration factor that converts detected laser light power to mirror displacement is measured by applying a known force to a test mass [43]. An auxiliary 1047-nm wavelength laser is reflected off the end test mass and modulated in intensity to generate a varying force. The response of the optical transducer is measured by sweeping the modulation frequency through the entire detection band. It is also tracked by a set of fixed frequency lines. This way, the calibrated strain readout is computed in real time with less than $10 \%$ uncertainty in amplitude. The overall variability of the sensitivity of the detector was about $\pm 10 \%$.

The main light source is a prestabilized 1064-nm wavelength Nd:YAG laser. It is followed by a high power amplifier stage, capable of generating a maximum output power of $180 \mathrm{~W}$ [44]. During the first observation run, only $20 \mathrm{~W}$ were injected into the interferometer. A triangular optical resonator of $32.9 \mathrm{~m}$ round-trip length is placed between the laser source and the interferometer to reject higher order transverse optical modes and to stabilize the laser frequency further [45]. At the output port, a bow-tie optical resonator of $1.3 \mathrm{~m}$ round-trip length is used to reject unwanted spatial and frequency components on the light. Optical curvature mismatch of the interferometer mirrors is caused by manufacturing imperfections and by thermal lensing due to heating from the main laser beam. A thermal compensation system provides active correction by means of ring heaters arranged around the test masses and a set of $\mathrm{CO}_{2}$ lasers for central heating [46].

The Advanced LIGO detector uses coupled optical resonators to maximize the sensitivity of the interferometric transducer. These optical resonators enhance the light power circulating in each arm while simultaneously optimizing the effective antenna length and the gravitationalwave signal bandwidth [47-51]. As the interferometer is held near a dark fringe, most of the light is reflected back to the laser source. Adding a partially transmissive mirror at the interferometer input forms an optical resonator, leading to a power gain of 35 to 40 at the beam splitter. The optical resonator in the interferometer arms enhances the circulating power by another factor of 300 . Thus, $20 \mathrm{~W}$ of laser power entering the interferometer results in nearly $100 \mathrm{~kW}$ circulating in each arm. A partially reflective mirror is also placed at the output port to enhance the signal extraction and to increase the detector bandwidth. The resulting differential pole frequency or detector bandwidth is $\simeq 335 \mathrm{~Hz}$ (H1) and $\simeq 390 \mathrm{~Hz}$ (L1) [19]. The optical mode matching in the output resonator is worse for $\mathrm{H} 1$.

All of these coupled optical resonators require servo controls to be brought and held on resonance [52]. The lengths of the optical resonators in the interferometer arms are stabilized to less than $100 \mathrm{fm}$, whereas the lengths of the other coupled resonators are kept within 1 to $10 \mathrm{pm}$ [19]. Similarly, the interferometer test masses are aligned within tens of nanoradians relative to the optical axis for optimal performance. The noise arising from sensing and control of these extra degrees of freedom are combined together in the curve labeled other DOF in Fig. 3. The Pound-Drever-Hall reflection locking technique is used to sense the auxiliary longitudinal degrees of freedom [53,54], while an interferometric wavefront sensing scheme is deployed for the alignment system [55,56]. The differential arm length is controlled by the same technique during lock acquisition, but switched to the offset locking technique described above when observing. Digital servo systems are used to feed control signals back to actuators which steer the relative longitudinal positions and orientations of the interferometer mirrors. To prevent reintroducing ground motion onto the test masses, electrostatic actuators [57] are mounted to a second quadruple pendulum known as the reaction chain. Only test masses use reaction chains; all other interferometer mirrors use coil actuators mounted on a rigid structure surrounding the suspensions.

Servo controls are also necessary for damping the plethora of normal modes of the pendular suspensions and for stabilizing the seismic isolation system to an inertial reference frame. Moreover, at high laser power, optical springs introduce angular instabilities due to photon radiation pressure-induced torques acting on the mirrors $[58,59]$, while the mirror acoustic modes introduce parametric instabilities [60,61]. At the current laser power, only one acoustic mode is unstable which can be tuned away by the ring heaters. Together with thermal heating, angular optical springs and multiple parametric instabilities are the main challenges that need to be overcome to increase the circulating laser power; both will require active damping for stable operations.

Overall, more than 300 digital control loops with bandwidths spanning from sub- $\mathrm{Hz}$ to hundreds of $\mathrm{kHz}$ are employed to keep each Advanced LIGO interferometer operating optimally during observation. The digital control computers also serve as the data acquisition system that continuously writes on the order of $10^{5}$ channels of time series data to disk, at a rate of $\simeq 12 \mathrm{Mbytes} / \mathrm{s}$. It is 
synchronized to GPS to better than $10 \mu \mathrm{s}$ [40]. A statebased automation controller provides hands-free running during operations.

Outlook.-The global gravitational-wave network will be significantly enhanced in the upcoming years. In 2016, Advanced LIGO will be joined by Advanced Virgo, the $3 \mathrm{~km}$ detector located near Pisa, Italy [62]. The Japanese KAGRA interferometer [63] and a possible third LIGO detector in India [64] will provide a global network that allows for improved parameter estimation and sky localization [65]. Achieving design sensitivity with the network of current detectors will define earthbound gravitationalwave astrophysics in the near future. Looking further ahead, we can envision current technologies leading to a factor of 2 improvement over the Advanced LIGO design sensitivity [13], so that events such as GW150914 could be detected with SNRs up to 200. More dramatic improvements will require significant technology development and new facilities.

The authors gratefully acknowledge the support of the United States National Science Foundation (NSF) for the construction and operation of the LIGO Laboratory and Advanced LIGO as well as the Science and Technology Facilities Council (STFC) of the United Kingdom, the Max-Planck-Society (MPS), and the State of Niedersachsen/ Germany for support of the construction of Advanced LIGO and construction and operation of the GEO600 detector. Additional support for Advanced LIGO was provided by the Australian Research Council. The authors gratefully acknowledge the Italian Istituto Nazionale di Fisica Nucleare (INFN), the French Centre National de la Recherche Scientifique (CNRS) and the Foundation for Fundamental Research on Matter supported by the Netherlands Organisation for Scientific Research, for the construction and operation of the Virgo detector and the creation and support of the EGO consortium. The authors also gratefully acknowledge research support from these agencies as well as by the Council of Scientific and Industrial Research of India, Department of Science and Technology, India, Science \& Engineering Research Board (SERB), India, Ministry of Human Resource Development, India, the Spanish Ministerio de Economía y Competitividad, the Conselleria d'Economia i Competitivitat and Conselleria d'Educació, Cultura i Universitats of the Govern de les Illes Balears, the National Science Centre of Poland, the European Commission, the Royal Society, the Scottish Funding Council, the Scottish Universities Physics Alliance, the Hungarian Scientific Research Fund (OTKA), the Lyon Institute of Origins (LIO), the National Research Foundation of Korea, Industry Canada and the Province of Ontario through the Ministry of Economic Development and Innovation, the Natural Science and Engineering Research Council Canada, Canadian Institute for Advanced Research, the Brazilian Ministry of Science, Technology, and Innovation, Russian Foundation for Basic Research, the
Leverhulme Trust, the Research Corporation, Ministry of Science and Technology (MOST), Taiwan and the Kavli Foundation. The authors gratefully acknowledge the support of the NSF, STFC, MPS, INFN, CNRS and the State of Niedersachsen/Germany for provision of computational resources. This document has been assigned the LIGO Laboratory Document No. LIGO-P1500237.

[1] B. P. Abbott et al. (LIGO Scientific Collaboration and Virgo Collaboration), Observation of Gravitational Waves from a Binary Black Hole Merger, Phys. Rev. Lett. 116, 061102 (2016).

[2] B. P. Abbott et al. (LIGO Scientific Collaboration and Virgo Collaboration), Properties of the binary black hole merger GW150914, arXiv:1602.03840.

[3] J. Aasi et al. (LIGO Scientific Collaboration), Advanced LIGO, Classical Quantum Gravity 32, 074001 (2015).

[4] B. P. Abbott et al. (LIGO Scientific Collaboration), LIGO: The laser interferometer gravitational-wave observatory, Rep. Prog. Phys. 72, 076901 (2009).

[5] T. Accadia et al. (Virgo Collaboration), Virgo: A laser interferometer to detect gravitational waves, J. Instrum. 7, P03012 (2012).

[6] B. P. Abbott et al. (LIGO Scientific Collaboration and Virgo Collaboration), Astrophysical implications of the binary black hole merger GW150914, Astrophys. J. Lett. 818, L22 (2016).

[7] B. P. Abbott et al. (LIGO Scientific Collaboration and Virgo Collaboration), Tests of general relativity with GW150914, arXiv:1602.03841.

[8] B. P. Abbott et al. (LIGO Scientific Collaboration and Virgo Collaboration), preceding Letter, GW150914: Implications for the Stochastic Gravitational-Wave Background from Binary Black Holes, Phys. Rev. Lett. 116, 131102 (2016).

[9] B. P. Abbott et al. (LIGO Scientific Collaboration, Virgo Collaboration, Australian Square Kilometer Array Pathfinder (ASKAP) Collaboration, BOOTES Collaboration, Dark Energy Survey and the Dark Energy Camera GWEM Collaborations, Fermi GBM Collaboration, Fermi LAT Collaboration, GRAvitational Wave Inaf TeAm (GRAWITA), INTEGRAL Collaboration, Intermediate Palomar Transient Factory (iPTF) Collaboration, The InterPlanetary Network, J-GEM Collaboration, La Silla-QUEST Survey, Liverpool Telescope Collaboration, Low Frequency Array (LOFAR) Collaboration, MASTER Collaboration, MAXI Collaboration, Murchison Wide-field Array (MWA) Collaboration, PanSTARRS Collaboration, PESSTO Collaboration, Pi of the Sky Collaboration, SkyMapper Collaboration, Swift Collaboration, TAROT, Zadko, Algerian National Observatory, and C2PU Collaboration, TOROS Collaboration and VISTA Collaboration), Localization and broadband follow-up of the gravitational-wave transient GW150914, arXiv:1602.08492.

[10] S. Adrián-Martínez et al. (ANTARES Collaboration, IceCube Collaboration, LIGO Scientific Collaboration, and Virgo Collaboration), High-energy neutrino follow-up search of gravitational wave event GW150914 with ANTARES and IceCube, arXiv:1602.05411. 
[11] K. S. Thorne, in 300 Years of Gravitation, edited by S. Hawking and W. Israel (Cambridge University Press, Cambridge, England, 1987), pp. 330-458.

[12] R. Weiss, Electromagnetically coupled broadband gravitational antenna, LIGO Report No. LIGO-P720002, https:// dcc.ligo.org/LIGO-P720002/public/main.

[13] J. Miller, L. Barsotti, S. Vitale, P. Fritschel, M. Evans, and D. Sigg, Prospects for doubling the range of Advanced LIGO, Phys. Rev. D 91, 062005 (2015).

[14] L. S. Finn, Binary inspiral, gravitational radiation, and cosmology, Phys. Rev. D 53, 2878 (1996).

[15] P. A. R. Ade et al. (Planck Collaboration), Planck 2013 results. XVI. Cosmological parameters, Astron. Astrophys. 571, A16 (2014).

[16] P. Ajith et al., Inspiral-Merger-Ringdown Waveforms for Black-Hole Binaries with Nonprecessing Spins, Phys. Rev. Lett. 106, 241101 (2011).

[17] B. P. Abbott et al. (LIGO Scientific Collaboration and Virgo Collaboration), Characterization of transient noise in Advanced LIGO relevant to gravitational wave signal GW150914, arXiv:1602.03844.

[18] B. P. Abbott et al. (LIGO Scientific Collaboration and Virgo Collaboration), The rate of binary black hole mergers inferred from Advanced LIGO observations surrounding GW150914, arXiv:1602.03842.

[19] B. P. Abbott et al. (LIGO Scientific Collaboration), The sensitivity of the Advanced LIGO detectors at the beginning of gravitational wave astronomy, LIGO Report No. LIGOP1500260 2016, https://dcc.ligo.org/LIGO-P1500260/ public/main.

[20] S. M. Aston et al., Update on quadruple suspension design for Advanced LIGO, Classical Quantum Gravity 29, 235004 (2012).

[21] V. B. Braginskiı̌, V. P. Mitrofanov, and O. A. Okhrimenko, Oscillators for free-mass gravitational antennas, JETP Lett. 55, 432 (1992).

[22] A. V. Cumming et al., Design and development of the advanced LIGO monolithic fused silica suspension, Classical Quantum Gravity 29, 035003 (2012).

[23] F. Matichard et al., Seismic isolation of Advanced LIGO: Review of strategy, instrumentation and performance, Classical Quantum Gravity 32, 185003 (2015).

[24] S. Wen et al., Hydraulic external pre-isolator system for LIGO, Classical Quantum Gravity 31, 235001 (2014).

[25] G. Cella, in Recent Developments in General Relativity, edited by B. Casciaro, D. Fortunato, M. Francaviglia, and A. Masiello (Springer, Milan, 2000), pp. 495-503.

[26] J. C. Driggers, J. Harms, and R. X. Adhikari, Subtraction of Newtonian noise using optimized sensor arrays, Phys. Rev. D 86, 102001 (2012).

[27] P. R. Saulson, Thermal noise in mechanical experiments, Phys. Rev. D 42, 2437 (1990).

[28] Y. Levin, Internal thermal noise in the LIGO test masses: A direct approach, Phys. Rev. D 57, 659 (1998).

[29] G. Harry, T. P. Bodiya, and R. DeSalvo, Optical Coatings and Thermal Noise in Precision Measurement (Cambridge University Press, Cambridge, England, 2012).

[30] G. M. Harry et al., Titania-doped tantala/silica coatings for gravitational-wave detection, Classical Quantum Gravity 24, 405 (2007).
[31] M. Granata et al., Mechanical loss in state-of-the-art amorphous optical coatings, Phys. Rev. D 93, 012007 (2016).

[32] R. Flaminio, J. Franc, C. Michel, N. Morgado, L. Pinard, and B. Sassolas, A study of coating mechanical and optical losses in view of reducing mirror thermal noise in gravitational wave detectors, Classical Quantum Gravity 27, 084030 (2010).

[33] J. Agresti, G. Castaldi, R. DeSalvo, V. Galdi, V. Pierro, and I. M. Pinto, Optimized multilayer dielectric mirror coatings for gravitational wave interferometers, Proc. SPIE Int. Soc. Opt. Eng. 6286, 628608 (2006).

[34] A. E. Villar et al., Measurement of thermal noise in multilayer coatings with optimized layer thickness, Phys. Rev. D 81, 122001 (2010).

[35] C. M. Caves, Quantum-Mechanical Radiation-Pressure Fluctuations in an Interferometer, Phys. Rev. Lett. 45, 75 (1980); Quantum-mechanical noise in an interferometer, Phys. Rev. D 23, 1693 (1981).

[36] V. B. Braginsky, F. Y. Khalili, and K. S. Thorne, Quantum Measurement (Cambridge University Press, Cambridge, England, 1992).

[37] D. E. McClelland, N. Mavalvala, Y. Chen, and R. Schnabel, Advanced interferometry, quantum optics and optomechanics in gravitational wave detectors, Laser Photonics Rev. 5, 677 (2011).

[38] A. Effler, R. M. S. Schofield, V. V. Frolov, G. González, K. Kawabe, J. R. Smith, J. Birch, and R. McCarthy, Environmental influences on the LIGO gravitational wave detectors during the 6th science run, Classical Quantum Gravity 32, 035017 (2015).

[39] T. T. Fricke et al., DC readout experiment in Enhanced LIGO, Classical Quantum Gravity 29, 065005 (2012).

[40] B. P. Abbott et al. (LIGO Scientific Collaboration), Calibration of the Advanced LIGO detectors for the discovery of the binary black-hole merger GW150914, arXiv: 1602.03845 .

[41] B. P. Abbott et al. (LIGO Scientific Collaboration and Virgo Collaboration), Observing gravitational-wave transient GW150914 with minimal assumptions, arXiv:1602.03843.

[42] B. P. Abbott et al. (LIGO Scientific Collaboration and Virgo Collaboration), GW150914: First results from the search for binary black hole coalescence with Advanced LIGO, arXiv:1602.03839.

[43] E. Goetz et al., Precise calibration of LIGO test mass actuators using photon radiation pressure, Classical Quantum Gravity 26, 245011 (2009).

[44] P. Kwee et al., Stabilized high-power laser system for the gravitational wave detector Advanced LIGO, Opt. Express 20, 10617 (2012).

[45] C. L. Mueller et al., The Advanced LIGO input optics, Rev. Sci. Instrum. 87, 014502 (2016).

[46] A.F. Brooks et al., Direct measurement of absorptioninduced wavefront distortion in high optical power systems, Appl. Opt. 48, 355 (2009).

[47] R. W. P. Drever, The Detection of Gravitational Waves, edited by D. G. Blair (Cambridge University Press, Cambridge, England, 1991) pp. 306.

[48] R. W. P. Drever et al., in Quantum Optics, Experimental Gravity, and Measurement Theory, NATO ASI Series B, 
Vol. 94, edited by P. Meystre and M. O. Scully (Plenum Press, New York, 1983), pp. 503-514.

[49] R. Schilling (private communication).

[50] B. J. Meers, Recycling in laser-interferometric gravitationalwave detectors, Phys. Rev. D 38, 2317 (1988).

[51] J. Mizuno, K. A. Strain, P. G. Nelson, J. M. Chen, R. Schilling, A. Rüdiger, W. Winkler, and K. Danzmann, Resonant sideband extraction: A new configuration for interferometric gravitational wave detectors, Phys. Lett. A 175, 273 (1993).

[52] A. Staley, D. Martynov et al., Achieving resonance in the Advanced LIGO gravitational-wave interferometer, Classical Quantum Gravity 31, 245010 (2014).

[53] R. W. P. Drever, J. L. Hall, F. V. Kowalski, J. Hough, G. M. Ford, A. J. Munley, and H. Ward, Laser phase and frequency stabilization using an optical resonator, Appl. Phys. B 31, 97 (1983).

[54] P. Fritschel, R. Bork, G. González, N. Mavalvala, D. Ouimette, H. Rong, D. Sigg, and M. Zucker, Readout and control of a power-recycled interferometric gravitational-wave antenna, Appl. Opt. 40, 4988 (2001).

[55] E. Morrison, B. J. Meers, D. I. Robertson, and H. Ward, Automatic alignment of optical interferometers, Appl. Opt. 33, 5041 (1994).

[56] L. Barsotti, M. Evans, and P. Fritschel, Alignment sensing and control in Advanced LIGO, Classical Quantum Gravity 27, 084026 (2010).

[57] C. Affeldt et al., Advanced techniques in GEO 600, Classical Quantum Gravity 31, 224002 (2014).
[58] J. A. Sidles and D. Sigg, Optical torques in suspended Fabry-Perot interferometers, Phys. Lett. A 354, 167 (2006).

[59] K. L. Dooley, L. Barsotti, R. X. Adhikari, M. Evans, T. T. Fricke, P. Fritschel, V. Frolov, K. Kawabe, and N. Smith-Lefebvre, Angular control of optical cavities in a radiation-pressure-dominated regime: The Enhanced LIGO case, J. Opt. Soc. Am. A 30, 2618 (2013).

[60] V. B. Braginsky, S. E. Strigin, and S. P. Vyatchanin, Analysis of parametric oscillatory instability in power recycled LIGO interferometer, Phys. Lett. A 305, 111 (2002).

[61] M. Evans et al., Observation of Parametric Instability in Advanced LIGO, Phys. Rev. Lett. 114, 161102 (2015).

[62] F. Acernese et al. (Virgo Collaboration), Advanced Virgo: A second-generation interferometric gravitational wave detector, Classical Quantum Gravity 32, 024001 (2015).

[63] Y. Aso, Y. Michimura, K. Somiya, M. Ando, O. Miyakawa, T. Sekiguchi, D. Tatsumi, and H. Yamamoto (KAGRA Collaboration), Interferometer design of the KAGRA gravitational wave detector, Phys. Rev. D 88, 043007 (2013).

[64] B. Iyer et al., LIGO-India Report No. LIGO-M1100296, 2011, https://dcc.ligo.org/LIGO-M1100296/public/main.

[65] B. P. Abbott et al. (LIGO Scientific Collaboration and Virgo Collaboration), Prospects for observing and localizing gravitational-wave transients with Advanced LIGO and Advanced Virgo, Living Rev. Relativity 19, 1 (2016).

B. P. Abbott, ${ }^{1}$ R. Abbott, ${ }^{1}$ T. D. Abbott, ${ }^{2}$ M. R. Abernathy, ${ }^{1}$ F. Acernese,,${ }^{3,43,4}$ K. Ackley, ${ }^{5}$ C. Adams, ${ }^{6}$ T. Adams, ${ }^{7}$ P. Addesso, ${ }^{3}$ R. X. Adhikari, ${ }^{1}$ V. B. Adya,${ }^{8}$ C. Affeldt,${ }^{8}$ M. Agathos, ${ }^{9}$ K. Agatsuma, ${ }^{9}$ N. Aggarwal, ${ }^{10}$ O. D. Aguiar, ${ }^{11}$ L. Aiello, ${ }^{12,13}$ A. Ain, ${ }^{14}$ P. Ajith, ${ }^{15}$ B. Allen, ${ }^{8,16,17}$ A. Allocca, ${ }^{18,19}$ P. A. Altin,${ }^{20}$ S. B. Anderson,,${ }^{1}$ W. G. Anderson, ${ }^{16}$ K. Arai, ${ }^{1}$ M. C. Araya, ${ }^{1}$ C. C. Arceneaux,${ }^{21}$ J. S. Areeda, ${ }^{22}$ N. Arnaud, ${ }^{23}$ K. G. Arun, ${ }^{24}$ S. Ascenzi,${ }^{25,13}$ G. Ashton,${ }^{26}$ M. Ast, ${ }^{27}$ S. M. Aston, ${ }^{6}$ P. Astone, ${ }^{28}$ P. Aufmuth ${ }^{8}$ C. Aulbert, ${ }^{8}$ S. Babak,${ }^{29}$ P. Bacon, ${ }^{30}$ M. K. M. Bader, ${ }^{9}$ P. T. Baker, ${ }^{31}$ F. Baldaccini, ${ }^{32,33}$ G. Ballardin, ${ }^{34}$ S. W. Ballmer, ${ }^{35}$ J. C. Barayoga, ${ }^{1}$ S. E. Barclay, ${ }^{36}$ B. C. Barish, ${ }^{1}$ D. Barker,,${ }^{37}$ F. Barone, ${ }^{3,4}$ B. Barr, ${ }^{36}$ L. Barsotti, ${ }^{10}$ M. Barsuglia,${ }^{30}$ D. Barta,${ }^{38}$ J. Bartlett, ${ }^{37}$ I. Bartos, ${ }^{39}$ R. Bassiri, ${ }^{40}$ A. Basti, ${ }^{18,19}$ J. C. Batch, ${ }^{37}$ C. Baune, ${ }^{8}$ V. Bavigadda,${ }^{34}$ M. Bazzan, ${ }^{41,42}$ B. Behnke, ${ }^{29}$ M. Bejger, ${ }^{43}$ A. S. Bell, ${ }^{36}$ C. J. Bell, ${ }^{36}$ B. K. Berger, ${ }^{1}$ J. Bergman, ${ }^{37}$ G. Bergmann, ${ }^{8}$ C. P. L. Berry, ${ }^{44}$ D. Bersanetti ${ }^{45,46}$ A. Bertolini, ${ }^{9}$ J. Betzwieser, ${ }^{6}$ S. Bhagwat,${ }^{35}$ R. Bhandare, ${ }^{47}$ I. A. Bilenko, ${ }^{48}$ G. Billingsley, ${ }^{1}$ J. Birch, ${ }^{6}$ R. Birney,${ }^{49}$ S. Biscans, ${ }^{10}$ A. Bisht,${ }^{8,17}$ M. Bitossi, ${ }^{34}$ C. Biwer, ${ }^{35}$ M. A. Bizouard ${ }^{23}$ J. K. Blackburn, ${ }^{1}$ C. D. Blair, ${ }^{50}$ D. G. Blair, ${ }^{50}$ R. M. Blair, ${ }^{37}$ S. Bloemen,${ }^{51}$ O. Bock, ${ }^{8}$ T. P. Bodiya,${ }^{10}$ M. Boer, ${ }^{52}$ G. Bogaert ${ }^{52}$ C. Bogan, ${ }^{8}$ A. Bohe,${ }^{29}$ P. Bojtos,${ }^{53}$ C. Bond,${ }^{44}$ F. Bondu, ${ }^{54}$ R. Bonnand, ${ }^{7}$ B. A. Boom, ${ }^{9}$ R. Bork, ${ }^{1}$ V. Boschi, ${ }^{18,19}$ S. Bose,${ }^{55,14}$ Y. Bouffanais, ${ }^{30}$ A. Bozzi,${ }^{34}$ C. Bradaschia, ${ }^{19}$ P. R. Brady, ${ }^{16}$ V. B. Braginsky, ${ }^{48}$ M. Branchesi,${ }^{56,57}$ J. E. Brau, ${ }^{58}$ T. Briant,${ }^{59}$ A. Brillet, ${ }^{52}$ M. Brinkmann, ${ }^{8}$ V. Brisson, ${ }^{23}$ P. Brockill, ${ }^{16}$ A. F. Brooks, ${ }^{1}$ D. A. Brown, ${ }^{35}$ D. D. Brown, ${ }^{44}$ N. M. Brown, ${ }^{10}$ C. C. Buchanan, ${ }^{2}$ A. Buikema, ${ }^{10}$ T. Bulik, ${ }^{60}$ H. J. Bulten, ${ }^{61,9}$ A. Buonanno, ${ }^{29,62}$ D. Buskulic, ${ }^{7}$ C. Buy, ${ }^{30}$ R. L. Byer, ${ }^{40}$

L. Cadonati, ${ }^{63}$ G. Cagnoli, ${ }^{64,65}$ C. Cahillane, ${ }^{1}$ J. Calderón Bustillo, ${ }^{66,63}$ T. Callister, ${ }^{1}$ E. Calloni, ${ }^{67,4}$ J. B. Camp, ${ }^{68}$ K. C. Cannon, ${ }^{69}$ J. Cao,${ }^{70}$ C. D. Capano, ${ }^{8}$ E. Capocasa, ${ }^{30}$ F. Carbognani,${ }^{34}$ S. Caride, ${ }^{71}$ J. Casanueva Diaz, ${ }^{23}$ C. Casentini, ${ }^{25,13}$ S. Caudill, ${ }^{16}$ M. Cavaglià, ${ }^{21}$ F. Cavalier ${ }^{23}$ R. Cavalieri, ${ }^{34}$ G. Cella, ${ }^{19}$ C. B. Cepeda, ${ }^{1}$ L. Cerboni Baiardi, ${ }^{56,57}$ G. Cerretani ${ }^{18,19}$ E. Cesarini, ${ }^{25,13}$ R. Chakraborty, ${ }^{1}$ T. Chalermsongsak, ${ }^{1}$ S. J. Chamberlin, ${ }^{72}$ M. Chan, ${ }^{36}$ S. Chao, ${ }^{73}$ P. Charlton,${ }^{74}$ E. Chassande-Mottin, ${ }^{30}$ H. Y. Chen, ${ }^{75}$ Y. Chen, ${ }^{76}$ C. Cheng, ${ }^{73}$ A. Chincarini, ${ }^{46}$ A. Chiummo, ${ }^{34}$ H. S. Cho, ${ }^{77}$ M. Cho, ${ }^{62}$ J. H. Chow ${ }^{20}$ N. Christensen, ${ }^{78}$ Q. Chu, ${ }^{50}$ S. Chua, ${ }^{59}$ S. Chung, ${ }^{50}$ G. Ciani, ${ }^{5}$ F. Clara, ${ }^{37}$ J. A. Clark ${ }^{63}$ F. Cleva, ${ }^{52}$ E. Coccia,${ }^{25,12,13}$ P.-F. Cohadon, ${ }^{59}$ A. Colla,${ }^{79,28}$ C. G. Collette, ${ }^{80}$ L. Cominsky, ${ }^{81}$ 
M. Constancio Jr., ${ }^{11}$ A. Conte, ${ }^{79,28}$ L. Conti, ${ }^{42}$ D. Cook,${ }^{37}$ T. R. Corbitt, ${ }^{2}$ N. Cornish, ${ }^{31}$ A. Corsi, ${ }^{71}$ S. Cortese,${ }^{34}$ C. A. Costa, ${ }^{11}$ M. W. Coughlin, ${ }^{78}$ S. B. Coughlin, ${ }^{82}$ J.-P. Coulon, ${ }^{52}$ S. T. Countryman, ${ }^{39}$ P. Couvares, ${ }^{1}$ E. E. Cowan, ${ }^{63}$ D. M. Coward ${ }^{50}$ M. J. Cowart, ${ }^{6}$ D. C. Coyne, ${ }^{1}$ R. Coyne, ${ }^{71}$ K. Craig, ${ }^{36}$ J. D. E. Creighton, ${ }^{16}$ J. Cripe, ${ }^{2}$ S. G. Crowder ${ }^{83}$ A. Cumming, ${ }^{36}$ L. Cunningham, ${ }^{36}$ E. Cuoco, ${ }^{34}$ T. Dal Canton, ${ }^{8}$ S. L. Danilishin, ${ }^{36}$ S. D'Antonio, ${ }^{13}$ K. Danzmann, ${ }^{17,8}$ N. S. Darman, ${ }^{84}$ V. Dattilo, ${ }^{34}$ I. Dave, ${ }^{47}$ H. P. Daveloza, ${ }^{85}$ M. Davier ${ }^{23}$ G. S. Davies,${ }^{36}$ E. J. Daw, ${ }^{86}$ R. Day,${ }^{34}$ D. DeBra, ${ }^{40}$ G. Debreczeni, ${ }^{38}$ J. Degallaix, ${ }^{65}$ M. De Laurentis, ${ }^{67,4}$ S. Deléglise, ${ }^{59}$ W. Del Pozzo, ${ }^{44}$ T. Denker, ${ }^{8,17}$ T. Dent, ${ }^{8}$ H. Dereli, ${ }^{52}$ V. Dergachev, ${ }^{1}$ R. T. DeRosa,${ }^{6}$ R. De Rosa,${ }^{67,4}$ R. DeSalvo, ${ }^{87}$ S. Dhurandhar, ${ }^{14}$ M. C. Díaz ${ }^{85}$ L. Di Fiore ${ }^{4}$ M. Di Giovanni, ${ }^{79,28}$ A. Di Lieto, ${ }^{18,19}$ S. Di Pace, ${ }^{79,28}$ I. Di Palma, ${ }^{29,8}$ A. Di Virgilio, ${ }^{19}$ G. Dojcinoski, ${ }^{88}$ V. Dolique ${ }^{65}$

F. Donovan, ${ }^{10}$ K. L. Dooley, ${ }^{21}$ S. Doravari, ${ }^{6,8}$ R. Douglas, ${ }^{36}$ T. P. Downes, ${ }^{16}$ M. Drago, ${ }^{8,89,90}$ R. W. P. Drever, ${ }^{1}$ J. C. Driggers, ${ }^{37}$ Z. Du, ${ }^{70}$ M. Ducrot,${ }^{7}$ S. E. Dwyer, ${ }^{37}$ T. B. Edo ${ }^{86}$ M. C. Edwards, ${ }^{78}$ A. Effler, ${ }^{6}$ H.-B. Eggenstein, ${ }^{8}$ P. Ehrens, ${ }^{1}$ J. Eichholz, ${ }^{5}$ S. S. Eikenberry, ${ }^{5}$ W. Engels, ${ }^{76}$ R. C. Essick, ${ }^{10}$ T. Etzel,,${ }^{1}$ M. Evans, ${ }^{10}$ T. M. Evans, ${ }^{6}$ R. Everett,${ }^{72}$ M. Factourovich, ${ }^{39}$ V. Fafone, ${ }^{25,13,12}$ H. Fair, ${ }^{35}$ S. Fairhurst, ${ }^{91}$ X. Fan, ${ }^{70}$ Q. Fang, ${ }^{50}$ S. Farinon, ${ }^{46}$ B. Farr, ${ }^{75}$ W. M. Farr, ${ }^{44}$ M. Favata ${ }^{88}$ M. Fays,${ }^{91}$ H. Fehrmann, ${ }^{8}$ M. M. Fejer, ${ }^{40}$ I. Ferrante, ${ }^{18,19}$ E. C. Ferreira, ${ }^{11}$ F. Ferrini, ${ }^{34}$ F. Fidecaro, ${ }^{18,19}$ I. Fiori,${ }^{34}$ D. Fiorucci, ${ }^{30}$ R. P. Fisher, ${ }^{35}$ R. Flaminio, ${ }^{65,92}$ M. Fletcher, ${ }^{36}$ J.-D. Fournier, ${ }^{52}$ S. Franco, ${ }^{23}$ S. Frasca, ${ }^{79,28}$ F. Frasconi, ${ }^{19}$ Z. Frei ${ }^{53}$ A. Freise, ${ }^{44}$ R. Frey, ${ }^{58}$ V. Frey,${ }^{23}$ T. T. Fricke, ${ }^{8}$ P. Fritschel, ${ }^{10}$ V. V. Frolov, ${ }^{6}$ P. Fulda,${ }^{5}$ M. Fyffe, ${ }^{6}$

H. A. G. Gabbard, ${ }^{21}$ J. R. Gair, ${ }^{93}$ L. Gammaitoni, ${ }^{32,33}$ S. G. Gaonkar, ${ }^{14}$ F. Garufi, ${ }^{67,4}$ A. Gatto, ${ }^{30}$ G. Gaur, ${ }^{94,95}$ N. Gehrels, ${ }^{68}$ G. Gemme, ${ }^{46}$ B. Gendre, ${ }^{52}$ E. Genin, ${ }^{34}$ A. Gennai, ${ }^{19}$ J. George,${ }^{47}$ L. Gergely, ${ }^{96}$ V. Germain, ${ }^{7}$ Archisman Ghosh, ${ }^{15}$ S. Ghosh,${ }^{51,9}$ J. A. Giaime, ${ }^{2,6}$ K. D. Giardina, ${ }^{6}$ A. Giazotto, ${ }^{19}$ K. Gill, ${ }^{97}$ A. Glaefke, ${ }^{36}$ E. Goetz, ${ }^{98}$ R. Goetz, ${ }^{5}$ L. Gondan,${ }^{53}$ G. González, ${ }^{2}$ J. M. Gonzalez Castro, ${ }^{18,19}$ A. Gopakumar, ${ }^{99}$ N. A. Gordon,${ }^{36}$ M. L. Gorodetsky, ${ }^{48}$ S. E. Gossan, ${ }^{1}$ M. Gosselin, ${ }^{34}$ R. Gouaty, ${ }^{7}$ C. Graef, ${ }^{36}$ P. B. Graff, ${ }^{62}$ M. Granata, ${ }^{65}$ A. Grant,,${ }^{36}$ S. Gras, ${ }^{10}$ C. Gray, ${ }^{37}$ G. Greco, ${ }^{56,57}$ A. C. Green ${ }^{44}$ P. Groot ${ }^{51}$ H. Grote ${ }^{8}$ S. Grunewald, ${ }^{29}$ G. M. Guidi,${ }^{56,57}$ X. Guo, ${ }^{70}$ A. Gupta, ${ }^{14}$ M. K. Gupta, ${ }^{95}$ K. E. Gushwa, ${ }^{1}$ E. K. Gustafson, ${ }^{1}$ R. Gustafson, ${ }^{98}$ J. J. Hacker, ${ }^{22}$ B. R. Hall ${ }^{55}$ E. D. Hall, ${ }^{1}$ G. Hammond, ${ }^{36}$ M. Haney, ${ }^{99}$ M. M. Hanke, ${ }^{8}$ J. Hanks, ${ }^{37}$ C. Hanna,${ }^{72}$ M. D. Hannam, ${ }^{91}$ J. Hanson, ${ }^{6}$ T. Hardwick, ${ }^{2}$ K. Haris, ${ }^{106}$ J. Harms,${ }^{56,57}$ G. M. Harry, ${ }^{100}$ I. W. Harry, ${ }^{29}$ M. J. Hart, ${ }^{36}$ M. T. Hartman, ${ }^{5}$ C.-J. Haster ${ }^{44}$ K. Haughian, ${ }^{36}$ A. Heidmann, ${ }^{59}$ M. C. Heintze,${ }^{5,6}$ H. Heitmann, ${ }^{52}$ P. Hello, ${ }^{23}$ G. Hemming, ${ }^{34}$ M. Hendry, ${ }^{36}$ I. S. Heng, ${ }^{36}$ J. Hennig, ${ }^{36}$ A. W. Heptonstall, ${ }^{1}$ M. Heurs, ${ }^{8,17}$ S. Hild, ${ }^{36}$ D. Hoak, ${ }^{101}$ K. A. Hodge, ${ }^{1}$ D. Hofman, ${ }^{65}$ S. E. Hollitt, ${ }^{102}$ K. Holt, ${ }^{6}$ D. E. Holz, ${ }^{75}$ P. Hopkins, ${ }^{91}$ D. J. Hosken, ${ }^{102}$ J. Hough, ${ }^{36}$ E. A. Houston, ${ }^{36}$ E. J. Howell,${ }^{50}$ Y. M. Hu, ${ }^{36}$ S. Huang,${ }^{73}$ E. A. Huerta, ${ }^{103,82}$ D. Huet, ${ }^{23}$ B. Hughey, ${ }^{97}$ S. Husa, ${ }^{66}$ S. H. Huttner, ${ }^{36}$ T. Huynh-Dinh, ${ }^{6}$ A. Idrisy, ${ }^{72}$ N. Indik, ${ }^{8}$ D. R. Ingram,${ }^{37}$ R. Inta, ${ }^{71}$ H. N. Isa, ${ }^{36}$ J.-M. Isac, ${ }^{59}$ M. Isi, ${ }^{1}$ G. Islas, ${ }^{22}$ T. Isogai, ${ }^{10}$ B. R. Iyer ${ }^{15} \mathrm{~K}$. Izumi,${ }^{37}$ T. Jacqmin,${ }^{59} \mathrm{H}$. Jang, ${ }^{77} \mathrm{~K}$. Jani,${ }^{63}$ P. Jaranowski, ${ }^{104}$ S. Jawahar, ${ }^{105} \mathrm{~F}$. Jiménez-Forteza, ${ }^{66}$ W. W. Johnson, ${ }^{2}$ D. I. Jones, ${ }^{26}$ R. Jones, ${ }^{36}$ R. J. G. Jonker, ${ }^{9}$ L. Ju, ${ }^{50}$ C. V. Kalaghatgi, ${ }^{24,91}$ V. Kalogera, ${ }^{82}$ S. Kandhasamy, ${ }^{21}$ G. Kang, ${ }^{77}$ J. B. Kanner, ${ }^{1}$ S. Karki, ${ }^{58}$ M. Kasprzack, $, 23,34$ E. Katsavounidis, ${ }^{10}$ W. Katzman, ${ }^{6}$ S. Kaufer, ${ }^{17}$ T. Kaur, ${ }^{50}$ K. Kawabe,${ }^{37}$ F. Kawazoe, ${ }^{8,17}$ F. Kéfélian, ${ }^{52}$ M. S. Kehl, ${ }^{69}$ D. Keitel, ${ }^{8,66}$ D. B. Kelley, ${ }^{35}$ W. Kells, ${ }^{1}$ R. Kennedy, ${ }^{86}$ J. S. Key, ${ }^{85}$ A. Khalaidovski, ${ }^{8}$ F. Y. Khalili, ${ }^{48}$ I. Khan, ${ }^{12}$ S. Khan, ${ }^{91}$ Z. Khan,${ }^{95}$ E. A. Khazanov, ${ }^{107}$ N. Kijbunchoo, ${ }^{37}$ C. Kim, ${ }^{77}$ J. Kim, ${ }^{108}$ K. Kim, ${ }^{109}$ Nam-Gyu Kim, ${ }^{77}$ Namjun Kim, ${ }^{40}$ Y.-M. Kim, ${ }^{108}$ E. J. King, ${ }^{102}$ P. J. King, ${ }^{37}$ D. L. Kinzel, ${ }^{6}$ J. S. Kissel, ${ }^{37}$ L. Kleybolte, ${ }^{27}$ S. Klimenko, ${ }^{5}$ S. M. Koehlenbeck, ${ }^{8}$ K. Kokeyama, ${ }^{2}$ S. Koley, ${ }^{9}$ V. Kondrashov, ${ }^{1}$ A. Kontos, ${ }^{10}$ M. Korobko, ${ }^{27}$ W. Z. Korth, ${ }^{1}$ I. Kowalska, ${ }^{60}$ D. B. Kozak, ${ }^{1}$ V. Kringel, ${ }^{8}$ A. Królak, ${ }^{110,111}$ C. Krueger, ${ }^{17}$ G. Kuehn, ${ }^{8}$ P. Kumar, ${ }^{69}$ L. Kuo ${ }^{73}$ A. Kutynia, ${ }^{110}$ B. D. Lackey, ${ }^{35}$ M. Landry, ${ }^{37}$ J. Lange, ${ }^{112}$ B. Lantz, ${ }^{40}$ P. D. Lasky, ${ }^{113}$ A. Lazzarini, ${ }^{1}$ C. Lazzaro, ${ }^{63,42}$ P. Leaci, ${ }^{29,79,28}$ S. Leavey, ${ }^{36}$ E. O. Lebigot, ${ }^{30,70}$ C. H. Lee, ${ }^{108}$ H. K. Lee, ${ }^{109}$ H. M. Lee, ${ }^{114}$ K. Lee, ${ }^{36}$ A. Lenon, ${ }^{35}$ M. Leonardi, ${ }^{89,90}$ J. R. Leong, ${ }^{8}$ N. Leroy, ${ }^{23}$ N. Letendre, ${ }^{7}$ Y. Levin, ${ }^{113}$ B. M. Levine, ${ }^{37}$ T. G. F. Li, ${ }^{1}$ A. Libson, ${ }^{10}$ T. B. Littenberg, ${ }^{115}$ N. A. Lockerbie, ${ }^{105}$ J. Logue, ${ }^{36}$ A. L. Lombardi, ${ }^{101}$ J. E. Lord, ${ }^{35}$ M. Lorenzini, ${ }^{12,13}$ V. Loriette, ${ }^{116}$ M. Lormand, ${ }^{6}$ G. Losurdo, ${ }^{57}$ J. D. Lough, ${ }^{8,17}$ H. Lück, ${ }^{17,8}$ A. P. Lundgren, ${ }^{8}$ J. Luo, ${ }^{78}$ R. Lynch, ${ }^{10}$ Y. Ma,${ }^{50}$ T. MacDonald, ${ }^{40}$ B. Machenschalk, ${ }^{8}$ M. MacInnis, ${ }^{10}$ D. M. Macleod, ${ }^{2}$ F. Magaña-Sandoval,${ }^{35}$ R. M. Magee ${ }^{55}$ M. Mageswaran, ${ }^{1}$ E. Majorana, ${ }^{28}$ I. Maksimovic, ${ }^{116}$ V. Malvezzi,${ }^{25,13}$ N. Man ${ }^{52}$ I. Mandel,${ }^{44}$ V. Mandic ${ }^{83}$ V. Mangano,${ }^{36}$ G. L. Mansell, ${ }^{20}$ M. Manske, ${ }^{16}$ M. Mantovani, ${ }^{34}$ F. Marchesoni, ${ }^{117,33}$ F. Marion, ${ }^{7}$ S. Márka, ${ }^{39}$ Z. Márka, ${ }^{39}$ A. S. Markosyan, ${ }^{40}$ E. Maros, ${ }^{1}$ F. Martelli, ${ }^{56,57}$ L. Martellini, ${ }^{52}$ I. W. Martin ${ }^{36}$ R. M. Martin, ${ }^{5}$ D. V. Martynov, ${ }^{1}$ J. N. Marx, ${ }^{1}$ K. Mason, ${ }^{10}$ A. Masserot, ${ }^{7}$ T. J. Massinger, ${ }^{35}$ M. Masso-Reid ${ }^{36}$ F. Matichard, ${ }^{10}$ L. Matone, ${ }^{39}$ N. Mavalvala, ${ }^{10}$ N. Mazumder, ${ }^{55}$ G. Mazzolo, ${ }^{8}$ R. McCarthy, ${ }^{37}$ D. E. McClelland ${ }^{20}$ S. McCormick,${ }^{6}$ S. C. McGuire, ${ }^{118}$ G. McIntyre, ${ }^{1}$ J. McIver, ${ }^{1}$ D. J. McManus, ${ }^{20}$ S. T. McWilliams, ${ }^{103}$ D. Meacher, ${ }^{72}$ G. D. Meadors,${ }^{29,8}$ J. Meidam, ${ }^{9}$ A. Melatos ${ }^{84}$ G. Mendell,,${ }^{37}$ D. Mendoza-Gandara, ${ }^{8}$ R. A. Mercer, ${ }^{16}$ E. Merilh, ${ }^{37}$ M. Merzougui, ${ }^{52}$ S. Meshkov, ${ }^{1}$ C. Messenger, ${ }^{36}$ C. Messick, ${ }^{72}$ P. M. Meyers, ${ }^{83}$ F. Mezzani, ${ }^{28,79}$ 
H. Miao, ${ }^{44}$ C. Michel,${ }^{65}$ H. Middleton, ${ }^{44}$ E. E. Mikhailov, ${ }^{119}$ L. Milano, ${ }^{67,4}$ J. Miller, ${ }^{10}$ M. Millhouse, ${ }^{31}$ Y. Minenkov, ${ }^{13}$ J. Ming, ${ }^{29,8}$ S. Mirshekari, ${ }^{120}$ C. Mishra, ${ }^{15}$ S. Mitra, ${ }^{14}$ V. P. Mitrofanov ${ }^{48}$ G. Mitselmakher, ${ }^{5}$ R. Mittleman, ${ }^{10}$ A. Moggi, ${ }^{19}$ M. Mohan, ${ }^{34}$ S. R. P. Mohapatra, ${ }^{10}$ M. Montani,${ }^{56,57}$ B. C. Moore,${ }^{88}$ C. J. Moore, ${ }^{121}$ D. Moraru, ${ }^{37}$ G. Moreno, ${ }^{37}$

S. R. Morriss ${ }^{85}$ K. Mossavi, ${ }^{8}$ B. Mours, ${ }^{7}$ C. M. Mow-Lowry, ${ }^{44}$ C. L. Mueller, ${ }^{5}$ G. Mueller, ${ }^{5}$ A. W. Muir, ${ }^{91}$ Arunava Mukherjee, ${ }^{15}$ D. Mukherjee, ${ }^{16}$ S. Mukherjee, ${ }^{85}$ N. Mukund,${ }^{14}$ A. Mullavey, ${ }^{6}$ J. Munch, ${ }^{102}$ D. J. Murphy,${ }^{39}$ P. G. Murray, ${ }^{36}$ A. Mytidis, ${ }^{5}$ I. Nardecchia, ${ }^{25,13}$ L. Naticchioni,${ }^{79,28}$ R. K. Nayak, ${ }^{122}$ V. Necula, ${ }^{5}$ K. Nedkova, ${ }^{101}$ G. Nelemans, ${ }^{51,9}$ M. Neri, ${ }^{45,46}$ A. Neunzert, ${ }^{98}$ G. Newton, ${ }^{36}$ T. T. Nguyen,${ }^{20}$ A. B. Nielsen,${ }^{8}$ S. Nissanke,${ }^{51,9}$ A. Nitz, ${ }^{8}$ F. Nocera, ${ }^{34}$ D. Nolting, ${ }^{6}$ M. E. Normandin, ${ }^{85}$ L. K. Nuttall, ${ }^{35}$ J. Oberling, ${ }^{37}$ E. Ochsner, ${ }^{16}$ J. O'Dell, ${ }^{123}$ E. Oelker, ${ }^{10}$ G. H. Ogin, ${ }^{124}$ J. J. Oh, ${ }^{125}$ S. H. Oh, ${ }^{125}$ F. Ohme, ${ }^{91}$ M. Oliver, ${ }^{66}$ P. Oppermann, ${ }^{8}$ Richard J. Oram, ${ }^{6}$ B. O’Reilly, ${ }^{6}$ R. O’Shaughnessy, ${ }^{112}$ D. J. Ottaway, ${ }^{102}$ R. S. Ottens, ${ }^{5}$ H. Overmier, ${ }^{6}$ B. J. Owen, ${ }^{71}$ A. Pai, ${ }^{106}$ S. A. Pai, ${ }^{47}$ J. R. Palamos, ${ }^{58}$ O. Palashov, ${ }^{107}$ C. Palomba, ${ }^{28}$ A. Pal-Singh, ${ }^{27}$ H. Pan, ${ }^{73}$ C. Pankow, ${ }^{82}$ F. Pannarale, ${ }^{91}$ B. C. Pant, ${ }^{47}$ F. Paoletti,${ }^{34,19}$ A. Paoli, ${ }^{34}$

M. A. Papa ${ }^{29,16,8}$ H. R. Paris, ${ }^{40}$ W. Parker, ${ }^{6}$ D. Pascucci, ${ }^{36}$ A. Pasqualetti, ${ }^{34}$ R. Passaquieti,${ }^{18,19}$ D. Passuello, ${ }^{19}$ B. Patricelli, ${ }^{18,19}$ Z. Patrick, ${ }^{40}$ B. L. Pearlstone ${ }^{36}$ M. Pedraza, ${ }^{1}$ R. Pedurand ${ }^{65}$ L. Pekowsky, ${ }^{35}$ A. Pele, ${ }^{6}$ S. Penn, ${ }^{126}$

A. Perreca, ${ }^{1}$ M. Phelps, ${ }^{36}$ O. Piccinni, ${ }^{79,28}$ M. Pichot,${ }^{52}$ F. Piergiovanni, ${ }^{56,57}$ V. Pierro, ${ }^{87}$ G. Pillant,${ }^{34}$ L. Pinard, ${ }^{65}$ I. M. Pinto ${ }^{87}$ M. Pitkin,${ }^{36}$ R. Poggiani, ${ }^{18,19}$ P. Popolizio, ${ }^{34}$ A. Post,${ }^{8}$ J. Powell,${ }^{36}$ J. Prasad,${ }^{14}$ V. Predoi,${ }^{91}$

S. S. Premachandra, ${ }^{113}$ T. Prestegard, ${ }^{83}$ L. R. Price, ${ }^{1}$ M. Prijatelj ${ }^{34}$ M. Principe,${ }^{87}$ S. Privitera, ${ }^{29}$ G. A. Prodi,${ }^{89,90}$ L. Prokhorov ${ }^{48}$ O. Puncken, ${ }^{8}$ M. Punturo, ${ }^{33}$ P. Puppo, ${ }^{28}$ M. Pürrer, ${ }^{29}$ H. Qi,${ }^{16}$ J. Qin, ${ }^{50}$ V. Quetschke,${ }^{85}$ E. A. Quintero, ${ }^{1}$ R. Quitzow-James, ${ }^{58}$ F. J. Raab, ${ }^{37}$ D. S. Rabeling, ${ }^{20}$ H. Radkins, ${ }^{37}$ P. Raffai,${ }^{53}$ S. Raja,${ }^{47}$ M. Rakhmanov, ${ }^{85}$ P. Rapagnani, ${ }^{79,28}$ V. Raymond, ${ }^{29}$ M. Razzano, ${ }^{18,19}$ V. Re, ${ }^{25}$ J. Read, ${ }^{22}$ C. M. Reed,${ }^{37}$ T. Regimbau ${ }^{52}$ L. Rei, ${ }^{46}$ S. Reid, ${ }^{49}$ D. H. Reitze, ${ }^{1,5}$ H. Rew, ${ }^{119}$ S. D. Reyes, ${ }^{35}$ F. Ricci, ${ }^{79,28}$ K. Riles, ${ }^{98}$ N. A. Robertson, ${ }^{1,36}$ R. Robie, ${ }^{36}$ F. Robinet, ${ }^{23}$ A. Rocchi, ${ }^{13}$ L. Rolland, J. G. Rollins, ${ }^{1}$ V. J. Roma ${ }^{58}$ R. Romano, ${ }^{3,4}$ G. Romanov, ${ }^{119}$ J. H. Romie, ${ }^{6}$ D. Rosińska, ${ }^{127,43}$ S. Rowan, ${ }^{36}$ A. Rüdiger, ${ }^{8}$ P. Ruggi, ${ }^{34}$ K. Ryan, ${ }^{37}$ S. Sachdev, ${ }^{1}$ T. Sadecki, ${ }^{37}$ L. Sadeghian, ${ }^{16}$ L. Salconi,${ }^{34}$ M. Saleem, ${ }^{106}$ F. Salemi, ${ }^{8}$ A. Samajdar, ${ }^{122}$ L. Sammut, ${ }^{84,113}$ E. J. Sanchez, ${ }^{1}$ V. Sandberg,${ }^{37}$ B. Sandeen, ${ }^{82}$ J. R. Sanders, ${ }^{98,35}$ B. Sassolas,${ }^{65}$ B. S. Sathyaprakash,${ }^{91}$ P. R. Saulson, ${ }^{35}$ O. Sauter, ${ }^{98}$ R. L. Savage, ${ }^{37}$ A. Sawadsky, ${ }^{17}$ P. Schale,${ }^{58}$ R. Schilling, ${ }^{8, \dagger}$ J. Schmidt, ${ }^{8}$ P. Schmidt, ${ }^{1,76}$ R. Schnabel, ${ }^{27}$ R. M. S. Schofield, ${ }^{58}$ A. Schönbeck, ${ }^{27}$ E. Schreiber, ${ }^{8}$ D. Schuette, ${ }^{8,17}$ B. F. Schutz,,${ }^{91,29}$ J. Scott, ${ }^{36}$ S. M. Scott, ${ }^{20}$ D. Sellers, ${ }^{6}$ A. S. Sengupta, ${ }^{94}$ D. Sentenac, ${ }^{34}$ V. Sequino, ${ }^{25,13}$ A. Sergeev, ${ }^{107}$ G. Serna, ${ }^{22}$ Y. Setyawati, ${ }^{51,9}$ A. Sevigny, ${ }^{37}$ D. A. Shaddock, ${ }^{20}$ S. Shah, ${ }^{51,9}$ M. S. Shahriar, ${ }^{82}$ M. Shaltev, ${ }^{8}$ Z. Shao, ${ }^{1}$ B. Shapiro, ${ }^{40}$ P. Shawhan, ${ }^{62}$ A. Sheperd ${ }^{16}$ D. H. Shoemaker ${ }^{10}$ D. M. Shoemaker,${ }^{63}$ K. Siellez, ${ }^{52,63}$ X. Siemens, ${ }^{16}$ D. Sigg, ${ }^{37}$ A. D. Silva, ${ }^{11}$ D. Simakov, ${ }^{8}$ A. Singer, ${ }^{1}$ L. P. Singer ${ }^{68}$ A. Singh, ${ }^{29,8}$ R. Singh, ${ }^{2}$ A. Singhal, ${ }^{12}$ A. M. Sintes, ${ }^{66}$ B. J. J. Slagmolen, ${ }^{20}$ J. R. Smith, ${ }^{22}$ N. D. Smith, ${ }^{1}$ R. J. E. Smith, ${ }^{1}$ E. J. Son, ${ }^{125}$ B. Sorazu, ${ }^{36}$ F. Sorrentino,${ }^{46}$ T. Souradeep ${ }^{14}$ A. K. Srivastava,${ }^{95}$ A. Staley, ${ }^{39}$ M. Steinke, ${ }^{8}$ J. Steinlechner, ${ }^{36}$ S. Steinlechner, ${ }^{36}$ D. Steinmeyer, ${ }^{8,17}$ B. C. Stephens, ${ }^{16}$ R. Stone,${ }^{85}$ K. A. Strain, ${ }^{36}$ N. Straniero, ${ }^{65}$ G. Stratta, ${ }^{56,57}$ N. A. Strauss, ${ }^{78}$ S. Strigin, ${ }^{48}$ R. Sturani, ${ }^{120}$ A. L. Stuver, ${ }^{6}$ T. Z. Summerscales, ${ }^{128}$ L. Sun, ${ }^{84}$ P. J. Sutton, ${ }^{91}$ B. L. Swinkels, ${ }^{34}$ M. J. Szczepańczyk, ${ }^{97}$ M. Tacca, ${ }^{30}$ D. Talukder,${ }^{58}$ D. B. Tanner,${ }^{5}$ M. Tápai, ${ }^{96}$ S. P. Tarabrin, ${ }^{8}$ A. Taracchini, ${ }^{29}$ R. Taylor, ${ }^{1}$ T. Theeg, ${ }^{8}$ M. P. Thirugnanasambandam, ${ }^{1}$ E. G. Thomas,${ }^{44}$ M. Thomas, ${ }^{6}$ P. Thomas, ${ }^{37}$ K. A. Thorne,${ }^{6}$ K. S. Thorne ${ }^{76}$ E. Thrane, ${ }^{113}$ S. Tiwari,${ }^{12}$ V. Tiwari,${ }^{91}$ K. V. Tokmakov, ${ }^{105}$ C. Tomlinson,${ }^{86}$ M. Tonelli, ${ }^{18,19}$ C. V. Torres, ${ }^{85, \$}$ C. I. Torrie, ${ }^{1}$ D. Töyrä, ${ }^{44}$ F. Travasso, ${ }^{32,33}$ G. Traylor, ${ }^{6}$ D. Trifirò, ${ }^{21}$ M. C. Tringali, ${ }^{89,90}$ L. Trozzo, ${ }^{129,19}$ M. Tse, ${ }^{10}$ M. Turconi, ${ }^{52}$ D. Tuyenbayev,${ }^{85}$ D. Ugolini, ${ }^{130}$ C. S. Unnikrishnan, ${ }^{99}$ A. L. Urban, ${ }^{16}$ S. A. Usman, ${ }^{35}$ H. Vahlbruch, ${ }^{17}$ G. Vajente, ${ }^{1}$ G. Valdes,${ }^{85}$ N. van Bakel,,${ }^{9}$ M. van Beuzekom,,${ }^{9}$ J. F. J. van den Brand,${ }^{61,9}$ C. Van Den Broeck, ${ }^{9}$ D. C. Vander-Hyde, ${ }^{35,22}$ L. van der Schaaf, ${ }^{9}$ J. V. van Heijningen, ${ }^{9}$ A. A. van Veggel, ${ }^{36}$ M. Vardaro, ${ }^{41,42}$ S. Vass, ${ }^{1}$ M. Vasúth, ${ }^{38}$ R. Vaulin, ${ }^{10}$ A. Vecchio,${ }^{44}$ G. Vedovato, ${ }^{42}$ J. Veitch,${ }^{44}$ P. J. Veitch,${ }^{102}$ K. Venkateswara, ${ }^{131}$ D. Verkindt, ${ }^{7}$ F. Vetrano, ${ }^{56,57}$ A. Viceré ${ }^{56,57}$ S. Vinciguerra, ${ }^{44}$ D. J. Vine, ${ }^{49}$ J.-Y. Vinet, ${ }^{52}$ S. Vitale, ${ }^{10}$ T. Vo, ${ }^{35}$ H. Vocca, ${ }^{32,33}$ C. Vorvick,${ }^{37}$ D. Voss, ${ }^{5}$ W. D. Vousden,${ }^{44}$ S. P. Vyatchanin, ${ }^{48}$ A. R. Wade,${ }^{20}$ L. E. Wade, ${ }^{132}$ M. Wade ${ }^{132}$ M. Walker, ${ }^{2}$ L. Wallace, ${ }^{1}$ S. Walsh, ${ }^{16,8,29}$ G. Wang, ${ }^{12}$ H. Wang, ${ }^{44}$ M. Wang, ${ }^{44}$ X. Wang, ${ }^{70}$ Y. Wang,${ }^{50}$ R. L. Ward, ${ }^{20}$ J. Warner, ${ }^{37}$ M. Was, ${ }^{7}$ B. Weaver, ${ }^{37}$ L.-W. Wei ${ }^{52}$ M. Weinert, ${ }^{8}$ A. J. Weinstein, ${ }^{1}$ R. Weiss, ${ }^{10}$ T. Welborn, ${ }^{6}$ L. Wen,${ }^{50}$ P. Weßels, ${ }^{8}$ T. Westphal, ${ }^{8}$ K. Wette, ${ }^{8}$ J. T. Whelan, ${ }^{112,8}$ S. E. Whitcomb, ${ }^{1}$ D. J. White, ${ }^{86}$ B. F. Whiting, ${ }^{5}$ R. D. Williams, ${ }^{1}$ A. R. Williamson, ${ }^{91}$ J. L. Willis, ${ }^{133}$ B. Willke, ${ }^{17,8}$ M. H. Wimmer, ${ }^{8,17}$ W. Winkler, ${ }^{8}$ C. C. Wipf, ${ }^{1}$ H. Wittel, ${ }^{8,17}$ G. Woan, ${ }^{36}$ J. Worden, ${ }^{37}$ J. L. Wright, ${ }^{36}$ G. Wu, ${ }^{6}$ J. Yablon, ${ }^{82}$ W. Yam,${ }^{10}$ H. Yamamoto, ${ }^{1}$ C. C. Yancey, ${ }^{62}$ M. J. Yap, ${ }^{20}$ H. Yu, ${ }^{10}$ M. Yvert, ${ }^{7}$ A. Zadrożny, ${ }^{10}$ L. Zangrando, ${ }^{42}$ 
M. Zanolin, ${ }^{97}$ J.-P. Zendri, ${ }^{42}$ M. Zevin, ${ }^{82}$ F. Zhang, ${ }^{10}$ L. Zhang, ${ }^{1}$ M. Zhang, ${ }^{119}$ Y. Zhang, ${ }^{112}$ C. Zhao, ${ }^{50}$ M. Zhou, ${ }^{82}$ Z. Zhou, ${ }^{82}$ X. J. Zhu, ${ }^{50}$ M. E. Zucker, ${ }^{1,10}$ S. E. Zuraw, ${ }^{101}$ and J. Zweizig ${ }^{1}$

(LIGO Scientific Collaboration and Virgo Collaboration)

${ }^{1}$ LIGO, California Institute of Technology, Pasadena, California 91125, USA

${ }^{2}$ Louisiana State University, Baton Rouge, Louisiana 70803, USA

${ }^{3}$ Università di Salerno, Fisciano, I-84084 Salerno, Italy

${ }^{4}$ INFN, Sezione di Napoli, Complesso Universitario di Monte Sant'Angelo, I-80126 Napoli, Italy

${ }^{5}$ University of Florida, Gainesville, Florida 32611, USA

${ }^{6}$ LIGO Livingston Observatory, Livingston, Louisiana 70754, USA

${ }^{7}$ Laboratoire d'Annecy-le-Vieux de Physique des Particules (LAPP), Université Savoie Mont Blanc, CNRS/IN2P3, F-74941 Annecy-le-Vieux, France

${ }^{8}$ Albert-Einstein-Institut, Max-Planck-Institut für Gravitationsphysik, D-30167 Hannover, Germany

${ }^{9}$ Nikhef, Science Park, 1098 XG Amsterdam, The Netherlands

${ }^{10}$ LIGO, Massachusetts Institute of Technology, Cambridge, Massachusetts 02139, USA

${ }^{11}$ Instituto Nacional de Pesquisas Espaciais, 12227-010 São José dos Campos, São Paulo, Brazil

${ }^{12}$ INFN, Gran Sasso Science Institute, I-67100 L'Aquila, Italy

${ }^{13}$ INFN, Sezione di Roma Tor Vergata, I-00133 Roma, Italy

${ }^{14}$ Inter-University Centre for Astronomy and Astrophysics, Pune 411007, India

${ }^{15}$ International Centre for Theoretical Sciences, Tata Institute of Fundamental Research, Bangalore 560012, India

${ }^{16}$ University of Wisconsin-Milwaukee, Milwaukee, Wisconsin 53201, USA

${ }^{17}$ Leibniz Universität Hannover, D-30167 Hannover, Germany

${ }^{18}$ Università di Pisa, I-56127 Pisa, Italy

${ }^{19}$ INFN, Sezione di Pisa, I-56127 Pisa, Italy

${ }^{20}$ Australian National University, Canberra, Australian Capital Territory 0200, Australia

${ }^{21}$ The University of Mississippi, University, Mississippi 38677, USA

${ }^{22}$ California State University Fullerton, Fullerton, California 92831, USA

${ }^{23}$ LAL, Université Paris-Sud, CNRS/IN2P3, Université Paris-Saclay, 91400 Orsay, France

${ }^{24}$ Chennai Mathematical Institute, Siruseri 603103, India

${ }^{25}$ Università di Roma Tor Vergata, I-00133 Roma, Italy

${ }^{26}$ University of Southampton, Southampton SO17 1BJ, United Kingdom

${ }^{27}$ Universität Hamburg, D-22761 Hamburg, Germany

${ }^{28}$ INFN, Sezione di Roma, I-00185 Roma, Italy

${ }^{29}$ Albert-Einstein-Institut, Max-Planck-Institut für Gravitationsphysik, D-14476 Potsdam-Golm, Germany

${ }^{30}$ APC, AstroParticule et Cosmologie, Université Paris Diderot, CNRS/IN2P3, CEA/Irfu, Observatoire de Paris,

Sorbonne Paris Cité, F-75205 Paris Cedex 13, France

${ }^{31}$ Montana State University, Bozeman, Montana 59717, USA

${ }^{32}$ Università di Perugia, I-06123 Perugia, Italy

${ }^{33}$ INFN, Sezione di Perugia, I-06123 Perugia, Italy

${ }^{34}$ European Gravitational Observatory (EGO), I-56021 Cascina, Pisa, Italy

${ }^{35}$ Syracuse University, Syracuse, New York 13244, USA

${ }^{36}$ SUPA, University of Glasgow, Glasgow G12 8QQ, United Kingdom

${ }^{37}$ LIGO Hanford Observatory, Richland, Washington 99352, USA

${ }^{38}$ Wigner RCP, RMKI, H-1121 Budapest, Konkoly Thege Miklós út 29-33, Hungary

${ }^{39}$ Columbia University, New York, New York 10027, USA

${ }^{40}$ Stanford University, Stanford, California 94305, USA

${ }^{41}$ Università di Padova, Dipartimento di Fisica e Astronomia, I-35131 Padova, Italy

${ }^{42}$ INFN, Sezione di Padova, I-35131 Padova, Italy

${ }^{43}$ CAMK-PAN, 00-716 Warsaw, Poland

${ }^{44}$ University of Birmingham, Birmingham B15 2TT, United Kingdom

${ }^{45}$ Università degli Studi di Genova, I-16146 Genova, Italy

${ }^{46}$ INFN, Sezione di Genova, I-16146 Genova, Italy

${ }^{47}$ RRCAT, Indore, Madhya Pradesh 452013, India

${ }^{48}$ Faculty of Physics, Lomonosov Moscow State University, Moscow 119991, Russia

${ }^{49}$ SUPA, University of the West of Scotland, Paisley PA1 2BE, United Kingdom

${ }^{50}$ University of Western Australia, Crawley, Western Australia 6009, Australia

${ }^{51}$ Department of Astrophysics/IMAPP, Radboud University Nijmegen, 6500 GL Nijmegen, The Netherlands

${ }^{52}$ Artemis, Université Côte d'Azur, CNRS, Observatoire Côte d'Azur, CS 34229, Nice cedex 4, France 
${ }^{53}$ MTA Eötvös University, “Lendulet” Astrophysics Research Group, Budapest 1117, Hungary

${ }^{54}$ Institut de Physique de Rennes, CNRS, Université de Rennes 1, F-35042 Rennes, France

${ }^{55}$ Washington State University, Pullman, Washington 99164, USA

${ }^{56}$ Università degli Studi di Urbino “Carlo Bo," I-61029 Urbino, Italy

${ }^{57}$ INFN, Sezione di Firenze, I-50019 Sesto Fiorentino, Firenze, Italy

${ }^{58}$ University of Oregon, Eugene, Oregon 97403, USA

${ }^{59}$ Laboratoire Kastler Brossel, UPMC-Sorbonne Universités, CNRS, ENS-PSL Research University, Collège de France, F-75005 Paris, France

${ }^{60}$ Astronomical Observatory Warsaw University, 00-478 Warsaw, Poland

${ }^{61}$ VU University Amsterdam, 1081 HV Amsterdam, The Netherlands

${ }^{62}$ University of Maryland, College Park, Maryland 20742, USA

${ }^{63}$ Center for Relativistic Astrophysics and School of Physics, Georgia Institute of Technology, Atlanta, Georgia 30332, USA

${ }^{64}$ Institut Lumière Matière, Université de Lyon, Université Claude Bernard Lyon 1, UMR CNRS 5306, 69622 Villeurbanne, France

${ }^{65}$ Laboratoire des Matériaux Avancés (LMA), IN2P3/CNRS, Université de Lyon, F-69622 Villeurbanne Lyon, France

${ }^{66}$ Universitat de les Illes Balears, IAC3-IEEC, E-07122 Palma de Mallorca, Spain

${ }^{67}$ Università di Napoli "Federico II," Complesso Universitario di Monte Sant'Angelo, I-80126 Napoli, Italy

${ }^{68}$ NASA/Goddard Space Flight Center, Greenbelt, Maryland 20771, USA

${ }^{69}$ Canadian Institute for Theoretical Astrophysics, University of Toronto, Toronto, Ontario M5S 3H8, Canada

${ }^{70}$ Tsinghua University, Beijing 100084, China

${ }^{71}$ Texas Tech University, Lubbock, Texas 79409, USA

${ }^{72}$ The Pennsylvania State University, University Park, Pennsylvania 16802, USA

${ }^{73}$ National Tsing Hua University, Hsinchu City, Taiwan 30013, Republic of China

${ }^{74}$ Charles Sturt University, Wagga Wagga, New South Wales 2678, Australia

${ }^{75}$ University of Chicago, Chicago, Illinois 60637, USA

${ }^{76}$ Caltech CaRT, Pasadena, California 91125, USA

${ }^{77}$ Korea Institute of Science and Technology Information, Daejeon 305-806, Korea

${ }^{78}$ Carleton College, Northfield, Minnesota 55057, USA

${ }^{79}$ Università di Roma “La Sapienza,” I-00185 Roma, Italy

${ }^{80}$ University of Brussels, Brussels 1050, Belgium

${ }^{81}$ Sonoma State University, Rohnert Park, California 94928, USA

${ }^{82}$ Northwestern University, Evanston, Illinois 60208, USA

${ }^{83}$ University of Minnesota, Minneapolis, Minnesota 55455, USA

${ }^{84}$ The University of Melbourne, Parkville, Victoria 3010, Australia

${ }^{85}$ The University of Texas Rio Grande Valley, Brownsville, Texas 78520, USA

${ }^{86}$ The University of Sheffield, Sheffield S10 2TN, United Kingdom

${ }^{87}$ University of Sannio at Benevento, I-82100 Benevento, Italy and INFN, Sezione di Napoli, I-80100 Napoli, Italy

${ }^{88}$ Montclair State University, Montclair, New Jersey 07043, USA

${ }^{89}$ Università di Trento, Dipartimento di Fisica, I-38123 Povo, Trento, Italy

${ }^{90}$ INFN, Trento Institute for Fundamental Physics and Applications, I-38123 Povo, Trento, Italy

${ }^{91}$ Cardiff University, Cardiff CF24 3AA, United Kingdom

${ }^{92}$ National Astronomical Observatory of Japan, 2-21-1 Osawa, Mitaka, Tokyo 181-8588, Japan

${ }^{93}$ School of Mathematics, University of Edinburgh, Edinburgh EH9 3FD, United Kingdom

${ }^{94}$ Indian Institute of Technology, Gandhinagar Ahmedabad, Gujarat 382424, India

${ }^{95}$ Institute for Plasma Research, Bhat, Gandhinagar 382428, India

${ }^{96}$ University of Szeged, Dóm tér 9, Szeged 6720, Hungary

${ }^{97}$ Embry-Riddle Aeronautical University, Prescott, Arizona 86301, USA

${ }^{98}$ University of Michigan, Ann Arbor, Michigan 48109, USA

${ }^{99}$ Tata Institute of Fundamental Research, Mumbai 400005, India

${ }^{100}$ American University, Washington, D.C. 20016, USA

${ }^{101}$ University of Massachusetts-Amherst, Amherst, Massachusetts 01003, USA

${ }^{102}$ University of Adelaide, Adelaide, South Australia 5005, Australia

${ }^{103}$ West Virginia University, Morgantown, West Virginia 26506, USA

${ }^{104}$ University of Biat ystok, 15-424 Biat ystok, Poland

${ }^{105}$ SUPA, University of Strathclyde, Glasgow G1 1XQ, United Kingdom

${ }^{106}$ IISER-TVM, CET Campus, Trivandrum, Kerala 695016, India

${ }^{107}$ Institute of Applied Physics, Nizhny Novgorod, 603950, Russia

${ }^{108}$ Pusan National University, Busan 609-735, Korea

${ }^{109}$ Hanyang University, Seoul 133-791, Korea

${ }^{110}$ NCBJ, 05-400 Świerk-Otwock, Poland

${ }^{111}$ IM-PAN, 00-956 Warsaw, Poland 
${ }^{112}$ Rochester Institute of Technology, Rochester, New York 14623, USA

${ }^{113}$ Monash University, Victoria 3800, Australia

${ }^{114}$ Seoul National University, Seoul 151-742, Korea

${ }^{115}$ University of Alabama in Huntsville, Huntsville, Alabama 35899, USA

${ }^{116}$ ESPCI, CNRS, F-75005 Paris, France

${ }^{117}$ Università di Camerino, Dipartimento di Fisica, I-62032 Camerino, Italy

${ }^{118}$ Southern University and A\&M College, Baton Rouge, Louisiana 70813, USA

${ }^{119}$ College of William and Mary, Williamsburg, Virginia 23187, USA

${ }^{120}$ Instituto de Física Teórica, University Estadual Paulista/ICTP South American Institute for Fundamental Research, São Paulo, São Paulo 01140-070, Brazil

${ }^{121}$ University of Cambridge, Cambridge CB2 1TN, United Kingdom

${ }^{122}$ IISER-Kolkata, Mohanpur, West Bengal 741252, India

${ }^{123}$ Rutherford Appleton Laboratory, HSIC, Chilton, Didcot, Oxon OX11 OQX, United Kingdom

${ }^{124}$ Whitman College, 345 Boyer Avenue, Walla Walla, Washington 99362 USA

${ }^{125}$ National Institute for Mathematical Sciences, Daejeon 305-390, Korea

${ }^{126}$ Hobart and William Smith Colleges, Geneva, New York 14456, USA

${ }^{127}$ Janusz Gil Institute of Astronomy, University of Zielona Góra, 65-265 Zielona Góra, Poland

${ }^{128}$ Andrews University, Berrien Springs, Michigan 49104, USA

${ }^{129}$ Università di Siena, I-53100 Siena, Italy

${ }^{130}$ Trinity University, San Antonio, Texas 78212, USA

${ }^{131}$ University of Washington, Seattle, Washington 98195, USA

${ }^{132}$ Kenyon College, Gambier, Ohio 43022, USA

${ }^{133}$ Abilene Christian University, Abilene, Texas 79699, USA

† Deceased, May 2015.

${ }^{*}$ Deceased, March 2015. 